\title{
Efficacy of dignity therapy for depression and anxiety in terminally ill patients: Early results of a randomized controlled trial
}

\author{
MIGUEL JULIÃO, M.D., ${ }^{1,2,3}$ ANTÓNIO BARBOSA, M.D., PH.D., ${ }^{1,4}$ FÁTIMA OLIVEIRA, R.N., ${ }^{3}$ \\ BALTAZAR NUNES, PH.D., ${ }^{5}$ AND ANTÓNIO VAZ CARNEIRO, M.D., PH.D. ${ }^{2}$ \\ ${ }^{1}$ Center of Bioethics \\ ${ }^{2}$ Center for Evidence Based Medicine, Faculty of Medicine of the University of Lisbon, Lisbon, Portugal \\ ${ }^{3}$ Department of Palliative Medicine, Instituto das Irmãs Hospitaleiras do Sagrado Coração de Jesus, Casa de Saúde da \\ Idanha, Belas, Portugal \\ ${ }^{4}$ Department of Psychiatry, Hospital de Santa Maria, Lisbon, Portugal \\ ${ }^{5}$ Department of Epidemiology, Instituto Nacional de Saúde Dr. Ricardo Jorge, Lisbon, Portugal \\ (RECEIVED June 9, 2012; ACCEPTED July 7, 2012)
}

\begin{abstract}
Objective: Dignity therapy (DT) is a short-term psychotherapy developed for patients living with a life-limiting illness. Our aim was to determine the influence of DT on symptoms of depression and anxiety in people with a life-threatening disease with high level of distress, referred to an inpatient palliative care unit.

Method: This was an open-label randomized controlled trial. Sixty terminally ill patients were randomly assigned to one of two groups: intervention group (DT + standard palliative care [SPC]) or control group (SPC alone). The main outcomes were symptoms of depression and anxiety, measured with the Hospital Anxiety and Depression Scale, assessed at baseline, day 4, day 15 , and day 30 of follow-up.

Results: Of the 60 participants, 29 were randomized to DTand 31 to SPC. Baseline characteristics were similar between the two groups. DT was associated with a significant decrease in depressive symptoms at day 4 and day 15 (mean $=-4.46,95 \% \mathrm{CI},-6.91-2.02, p=0.001 ;$ mean $=-3.96,95 \%$ $\mathrm{CI},-7.33$ to $-0.61 ; p=0.022$, respectively), but not at day 30 (mean $=-3.33,95 \% \mathrm{CI},-7.32-0.65$, $p=0.097)$. DTwas also associated with a significant decrease in anxiety symptoms at each follow-up (mean $=-3.96,95 \% \mathrm{CI},-6.66$ to $-1.25, p=0.005$; mean $=-6.19,95 \% \mathrm{CI},-10.49$ to $-1.88, p=$ 0.006 ; mean $=-5.07,95 \%$ CI, -10.22 to $-0.09, p=0.054$, respectively).

Significance of results: DT appears to have a short-term beneficial effect on the depression and anxiety symptoms that often accompany patients at the end of their lives. Future research with larger samples compared with other treatments is needed to better understand the potential benefits of this psychotherapy.
\end{abstract}

KEYWORDS: DT, Depression and anxiety symptoms, Randomized controlled trial

\section{INTRODUCTION}

Terminally ill patients are often confronted with psychological distress, which is one of the most debilitating conditions among dying patients (Boston et al., 2011). The diagnosis of an incurable disease

Address correspondence to: Miguel Julião, Center of Bioethics, Av. Prof. Egas Moniz, 1649-028 Lisbon, Portugal. E-mail: migueljuliao@gmail.com can set patients into crisis, leaving them vulnerable to depression and anxiety. Although psychological distress is well documented in dying patients, it tends to be under-recognized and frequently left unattended. This accentuates patient and family suffering, reducing quality of life and the ability to connect with one another or come to terms with impending losses (Block, 2000).

Depression is the most common psychological problem in the terminally ill and is known as a risk 
factor for suicide and requests for hastened death (Chochinov et al., 1995). Terminally ill patients can face anxiety as an isolated psychiatric disorder or as a prominent component of acute or chronic physical symptoms such as pain or dyspnea.

Psychological suffering for terminally ill patients is also often framed in terms of loss of dignity (Chochinov, 2007). Although dignity is a core element of palliative care, it was not well defined or operationalized until the work of Chochinov and colleagues. Their Model of Dignity provides an empirical framework, offering insights regarding how patients face terminal illness. This model provides the theoretical basis for a brief, individualized intervention designed to address psychosocial and existential distress among terminally ill patients, termed dignity therapy (DT) (Chochinov et al., 2005).

Previous results conducted with patients with advanced cancer showed that, after performing DT, $91 \%$ of participants reported being satisfied; $76 \%$ reported a heightened sense of dignity; $68 \%$ reported an increased sense of purpose; $67 \%$ reported a heightened sense of meaning; and $47 \%$ reported an increased will to live (Chochinov et al., 2005). A recent trial of DT showed that although DT did not significantly influence distress, those assigned to DT where significantly more likely than other study groups to find this approach helpful, and to experience improved quality of life and an increased sense of dignity. DT also changed how their family saw and appreciated them, and was helpful to their family (Chochinov et al., 2011). Because base rates of distress were low in this study, DT did not show any effect on patientś depression and anxiety symptoms. As such, the authors stressed the importance of future research among more distressed patients to examine its ability to mitigate outright distress. Prior studies have shown that Portuguese cancer patients demonstrate high levels of depression and anxiety symptoms as measured by the Hospital Anxiety and Depression Scale (HADS) (Pais-Ribeiro et al., 2007).

The overall study objective was to provide data on several psychosocial distress outcomes such as desire for death, demoralization, loss of dignity, and depression and anxiety symptoms. The present article reports only the effect of DT on depression and anxiety symptoms immediately post-intervention, and at 15 and 30 day follow-up, in people with a life-threatening disease being cared for within an inpatient palliative medicine unit in Lisbon.

\section{METHOD}

This was an open-label randomized controlled trial composed of two study arms: an intervention group, consisting of DT in addition to standard palliative care (SPC); and a control group, consisting of SPC alone, with several evaluation assessments using the HADS according to the investigation protocol (Table 1).

\section{Participants}

Patients were recruited from a 10 bed specialized palliative medicine unit of S. Bento Menni in Lisbon that delivers differentiated palliative care to terminally ill patients from the Portuguese National Palliative Care Network. The recruitment took place over a 22 month period (from May 2010 to March 2012).

Inclusion criteria included: age $\geq 18$ years old; having a life-threatening disease with life expectancy of $\leq 6$ months or less; no evidence of dementia or delirium, based on documentation within the medical chart or by clinical consensus; Mini Mental State score $\geq 20$; being able to read and speak Portuguese; provision of written informed consent; and being available for four to five research encounters, over a period of 1 month.

This study was approved by the Ethics Committee of the Instituto das Irmãs Hospitaleiras do Sagrado Coração de Jesus - Casa de Saúde da Idanha.

\section{Randomization and Masking}

The randomization process was computer generated, using a fixed block of four, and was conducted by an independent statistician. Allocation concealment was made by using sequentially numbered envelopes for consecutive trial participants. After the baseline measures were collected by the secondary investigator (FO), the envelope was opened in the presence of the patient to reveal which study arm the patient was assigned to. The principal investigator (MJ) was not blind to patientś study groups, but was blind to the psychometric results throughout the entire protocol. The secondary investigator was blind to the content of DT sessions.

\section{Interventions}

$S P C$

SPC was provided by a multiprofessional palliative medicine team, including three palliative care physicians (including the principal investigator), nurses, a psychologist, and psychosocial worker. Clinical care consisted of daily (median $=25$ minutes; range 10-30) clinical interviews, physical examinations, and symptom assessment and management. Throughout their hospital stay, all patient and family questions were responded to, and existential and psychosocial support were offered. SPC was provided throughout the patients' hospital stay, including the duration of time they were enrolled within this study. As was the case with every other patient admitted to 
Table 1. Investigation protocol for each group.

\begin{tabular}{|c|c|c|c|c|c|c|c|c|c|}
\hline $\begin{array}{l}\text { Evaluation } \\
\text { time points }\end{array}$ & T0 & $\mathrm{T} 1$ & & & & & $\mathrm{~T} 2$ & $\begin{array}{c}\text { Follow-up } \\
\text { T3 }\end{array}$ & $\mathrm{T} 4$ \\
\hline $\begin{array}{l}\text { Days } \\
\text { Investigator } \\
\text { Protocol } \\
\quad \text { measure \& } \\
\text { assessment } \\
\text { instruments }\end{array}$ & \begin{tabular}{c}
\multicolumn{1}{c}{1} \\
FO \\
- Collection of \\
clinical and \\
demographic \\
data
\end{tabular} & \begin{tabular}{l}
\multicolumn{1}{c}{2} \\
FO \\
- Baseline \\
assessment: \\
- HADS \\
- Explanation \\
of DT and \\
delivery of \\
DT questions
\end{tabular} & 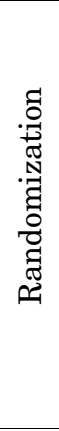 & 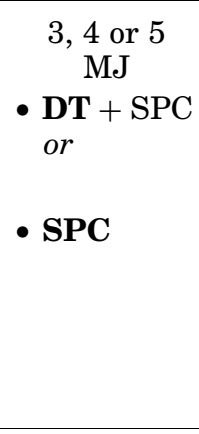 & 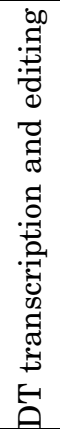 & $\begin{array}{l}3 \text { (post-DT) } \\
\text { MJ } \\
\text { - Review and } \\
\text { delivery of } \\
\text { generativity } \\
\text { document }\end{array}$ & $\begin{array}{l}4 \text { (post-DT) } \\
\quad \text { FO } \\
\text { - Follow-up } \\
\text { assessment: } \\
\text { - HADS }\end{array}$ & $\begin{array}{l}15 \text { (post-DT) } \\
\text { FO } \\
\text { - Follow-up } \\
\text { assessment: } \\
\text { - HADS }\end{array}$ & $\begin{array}{l}30 \text { (post-DT) } \\
\text { FO } \\
\text { - Follow-up } \\
\text { assessment: } \\
\text { - HADS }\end{array}$ \\
\hline
\end{tabular}

DT $=$ dignity therapy. HADS $=$ Hospital Anxiety and Depression Scale. SPC $=$ standard palliative care. 
this unit, they were seen and managed by the entire multiprofessional team.

\section{$D T$}

DT is a brief psychotherapeutic approach with the aim of bolstering the patient's sense of meaning and purpose, reinforcing a continued sense of worth within a framework that is supportive, nurturing, and accessible for those near death. Patients enrolled in DT are asked to audiorecord aspects of their lives that they would most want their loved ones to know about or remember. DT offers patients the opportunity to talk about issues that matter the most to them, to share moments that they feel were the most important and meaningful; to speak about things that they would like to be remembered by, or offer advice to their family and friends. These recorded sessions provide the basis of an edited transcript - generativity document - that is returned to patients for them to share with individuals of their choosing. Creating this document is meant to make patients feel valued, and that something of themselves will last beyond death.

Therapeutic sessions, running between 30 and 60 minutes, were offered at patientś bedsides and audiorecorded. Every tape-recorded session was erased after the completion of the study protocol. This study utilized the DT question framework (Chochinov et al., $2002 ; 2005)$, as per previously published trials (Table 2). All DTwas conducted by the principal investigator (MJ) who attended an International DT workshop held in Winnipeg, Canada; all procedures for DT were conducted in accordance with the DT Manual (Chochinov et al., 2002; 2005; Chochinov, 2011)

Patients meeting eligibility criteria and agreeing to participate in the study were asked to provide written informed consent. Once patients completed the baseline assessment (T1), those randomized to DT were provided with the standard framework of questions, thus giving them time to reflect and shape their eventual responses (Table 1). The taped DT session was scheduled to take place within 2-3 days, or as soon as the patient was available. Guided by the DT framework, the therapists followed the patients' cues, helping them to structure and organize their thoughts. Once the taped session was complete, over the course of the next $2-3$ days, the patients' recorded dialogue was transcribed verbatim and then edited (as per the DT protocol/manual) and reshaped into a written narrative. Once this editing process was complete, another session was arranged as soon as possible, for the therapist to read the document to the patient, allowing for final editorial corrections and revisions. The final version of the generativity document was given to patients to be passed along to the individuals of their choosing.
Table 2. Dignity therapy question protocol

Tell me a little about your life history; particularly the parts that you either remember most or think are the most important? When did you feel most alive?

Are there particular things that you would want your family to know about you, and are there particular things you would want them to remember?

What are the most important roles you have played in your life (family roles, vocational roles, community service roles, etc)? Why were they so important to you, and what do you think you accomplished within those roles?

What are your most important accomplishments, and what do you feel most proud of?

Are there particular things that you feel still need to be said to your loved ones, or things that you would want to take the time to say once again?

What are your hopes and dreams for your loved ones?

What have you learned about life that you would want to pass along to others? What advice or words of guidance would you wish to pass along to your [son, daughter, husband, wife, parents, other(s)]?

Are there words or perhaps even instructions you would like to offer your family, in order to provide them with comfort or solace?

In creating this permanent record, are there other things that you would like included?

\section{Outcomes}

The outcome evaluated was the influence of DT on depression and anxiety symptoms, measured with the HADS, at baseline (T1), day 4 (T2), day 15 (T3,) and day 30 (T4) of follow-up (Table 1). The HADS final scores were known only at the end of the completion of the study protocol of each patient.

\section{Statistical Analysis}

The data were analyzed with the SPSS ${ }^{\circledR} 18.0$ statistical package.

Based on previously published Portuguese studies of the HADS in cancer populations (Pais-Ribeiro et al., 2007), we considered a mean reduction of 1.5 points on the HADS depression subscale as clinically significant. Assuming a type I error of $5 \%$, a power of $80 \%$, and a standard deviation of 2.5 , each study arm required at least 45 patients. Assuming a dropout rate of $10 \%$, we intended to include in the overall study 50 patients in each group.

Our study protocol included two analyses: an interim analysis after 60 participants were obtained and another analysis at the end of the research (predicted year 2013). Therefore, this report includes only the efficacy data on depression and anxiety symptoms that were available by March 2012, for the first 60 who completed the protocol.

We compared continuous variables and ordinal rating scales using paired or independent $t$ test as 
appropriate. The criterion for statistical significance was set at $p<0.05$ in a two-tailed test. In order to check the $t$ test assumption of normality, we used the Shapiro-Wilk test. Nonparametric tests (Mann-Whitney test for comparisons between groups and Wilcoxon test for comparisons within groups) were also applied to validate the results obtained with $t$ tests. The statistical analysis was applied to all the patients who had at least one complete evaluation at any given follow-up point (T2, T3, T4 - per protocol analysis).

This study is registered with http://www.controlled-trials.com/ISRCTN34354086.

\section{RESULTS}

Summary demographic and illness data for both groups are presented in Table 3. Baseline characteristics of

Table 3. Summary characteristics of the participants at baseline $(\mathrm{n}=60)$

\begin{tabular}{|c|c|c|}
\hline & Control group $(n=31)$ & DT group $(n=29)$ \\
\hline \multicolumn{3}{|l|}{ Gender, $n(\%)$} \\
\hline Male & $13(41.9)$ & $14(48.3)$ \\
\hline Female & $18(58.1)$ & $15(51.7)$ \\
\hline \multicolumn{3}{|l|}{ Age, years } \\
\hline mean (SD) & \multicolumn{2}{|c|}{$66.5(12.4)$, range $=41-90$} \\
\hline$\leq 65$ & $13(41.9)$ & $17(58.6)$ \\
\hline$>65$ & $18(58.1)$ & $12(41.4)$ \\
\hline \multicolumn{3}{|l|}{ Race/Ethnicity, $n(\%)$} \\
\hline Caucasian & $29(93.5)$ & $28(96.5)$ \\
\hline African & $1(6.5)$ & $1(3.5)$ \\
\hline \multicolumn{3}{|l|}{ Marital status, $n(\%)$} \\
\hline Single & $6(19.4)$ & $5(17.2)$ \\
\hline Married/ Common law & $13(41.9)$ & $15(51.7)$ \\
\hline Divorced/separated & $3(9.7)$ & $4(13 \cdot 8)$ \\
\hline Widowed & $9(29.0)$ & $5(17.2)$ \\
\hline \multicolumn{3}{|l|}{ Education, $n(\%)$} \\
\hline Knows how to read and write (without instruction) & $1(3.2)$ & $2(6.9)$ \\
\hline Primary school & $14(45.2)$ & $10(34.5)$ \\
\hline High school & $10(32.2)$ & $10(34.5)$ \\
\hline Licensure & $6(19.4)$ & $7(24.1)$ \\
\hline \multicolumn{3}{|l|}{ Religion, $n(\%)$} \\
\hline Catholic & $26(83.9)$ & $26(89.7)$ \\
\hline Other & $5(16.1)$ & $3(10.3)$ \\
\hline \multicolumn{3}{|l|}{ Diagnosis, $n(\%)$} \\
\hline Cancer ${ }^{a}$ & $29(93.5)$ & $28(96.5)$ \\
\hline Non-cancer ${ }^{\mathrm{b}}$ & $2(6.5)$ & $1(3.5)$ \\
\hline \multicolumn{3}{|l|}{ Time since diagnosis ${ }^{\mathbf{c}}, n(\%)$} \\
\hline$<1$ year & $2(6.5)$ & $4(13.8)$ \\
\hline $1-2$ years & $11(35.5)$ & $5(17.2)$ \\
\hline$\geq 2$ years & $18(58.0)$ & $20(69.0)$ \\
\hline \multicolumn{3}{|l|}{ Psychiatric drugs, $n(\%)$} \\
\hline Antidepressants & $17(54.8)$ & $18(62.1)$ \\
\hline Anxiolytics & $16(51.6)$ & $15(51.7)$ \\
\hline Neuroleptics & $0(0)$ & $1(3.5)$ \\
\hline Anticonvulsivants & $10(32.3)$ & $11(37.9)$ \\
\hline \multicolumn{3}{|l|}{ Palliative Performance Scale d $^{d}$} \\
\hline & \multicolumn{2}{|c|}{$54.8(16.9)$, range $=30-90$} \\
\hline \multirow{3}{*}{$\begin{array}{l}\text { mean (SD) } \\
\text { Mini Mental State }\end{array}$} & $55.5(18.0)$ & $54.1(15.9)$ \\
\hline & \multirow{2}{*}{\multicolumn{2}{|c|}{$24.1(2.0)$, range $=20-28$}} \\
\hline & & \\
\hline Mean (SD) & $23.8(1.9)$ & $24.4(2.1)$ \\
\hline
\end{tabular}

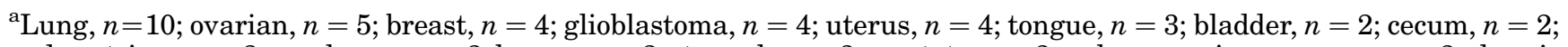
endometrium, $n=2$; esophagus, $n=2$; larynx, $n=2$; stomach, $n=2$; prostate, $n=2$; unknown primary cancer, $n=2$; chronic myeloid leukemia, $n=1$; dorsal neurinoma, $n=1$; glioma, $n=1$;melanoma, $n=1$; neoplasm of the nose, $n=1$; pancreas, $n=$ 1; small bowel neuroendocrine tumor, $n=1$; vascular arterial cancer, $n=1$.

${ }^{\mathrm{b}}$ Lateral amyotrophic sclerosis, $n=2$; trigeminal neuralgia, $n=1$.

${ }^{\mathrm{c}}$ Based on medical records or patients information.

${ }^{\mathrm{d}}$ Palliative Performance Scale: $100 \%$ - healthy; 0\% - death.

DT, dignity therapy. 
the patients were similar between the two groups. Over a 22 month period, 127 patients were admitted into the palliative care unit ward; 70 patients were assessed for eligibility, 60 of whom were randomized (29 to DT and 31 to SPC) (Fig. 1). After randomization, one patient died before receiving DT and another one presented severe clinical deterioration; six patients were lost to follow-up at T2 (DT: $n=2$; SPC: $n=4$ ); 15 patients were lost to follow-up at T3 (DT: $n=7$; SPC: $n=6$ ) and an additional nine were lost to follow-up at T4 (DT: $n=3$; SPC: $n=6)$ (Fig. 1).

The mean survival time was 26.8 days (DT $=25.8$, $\mathrm{SPC}=27.8 ; p=0.385$ ).

\section{Depression}

Both groups had a mean score (SD) on the HADS depression subscale $>11$ at baseline (SPC:13.37 [4.06]; DT:12.90 [4.80]; $p=0.708$ ).

Patients assigned to DT demonstrated a significant decrease on depression ratings at day 4 and at day 15 (mean $=-2.76,95 \% \mathrm{CI},-4.86$ to -0.66 , $p=0.012$; mean $=-1.44,95 \%$ CI $,-4.53-1.64, p=$ 0.034 , respectively), which plateaued by day 30 assessments $($ mean $=0.06,95 \%$ CI $,-3.65-3.78, p=$ 0.970) (Table 4).

Compared with the SPC group, those receiving DT demonstrated a significant reduction in depression ratings between baseline and day 4 assessment $($ mean $=-4.46,95 \%$ CI, -6.91 to $-2.02, p=$ 0.001 ); these differences across groups were maintained at day 15 (mean $=-3.96,95 \% \mathrm{CI},-7.33$ to $-0.61, p=0.022$ ) but not at day 30 (mean $=$ $-3.33,95 \%$ CI, $-7.32-0.65, p=0.097$ ) (Table 4).

Within the SPC group, there was a significant increase in depression ratings between baseline and all subsequent follow-up assessments (mean $=1.70$, $95 \%$ CI, $0.35-3.01, p=0.016$; mean $=2.52,95 \%$ CI, $1.02-4.03, p=0.002$; mean $=3.40$, 95\% CI, $1.66-$ $5.14, p=0.001$, respectively) (Table 4, Fig. 2).

The $t$ test normality assumption was rejected for the time measures of HADS depression subscale at

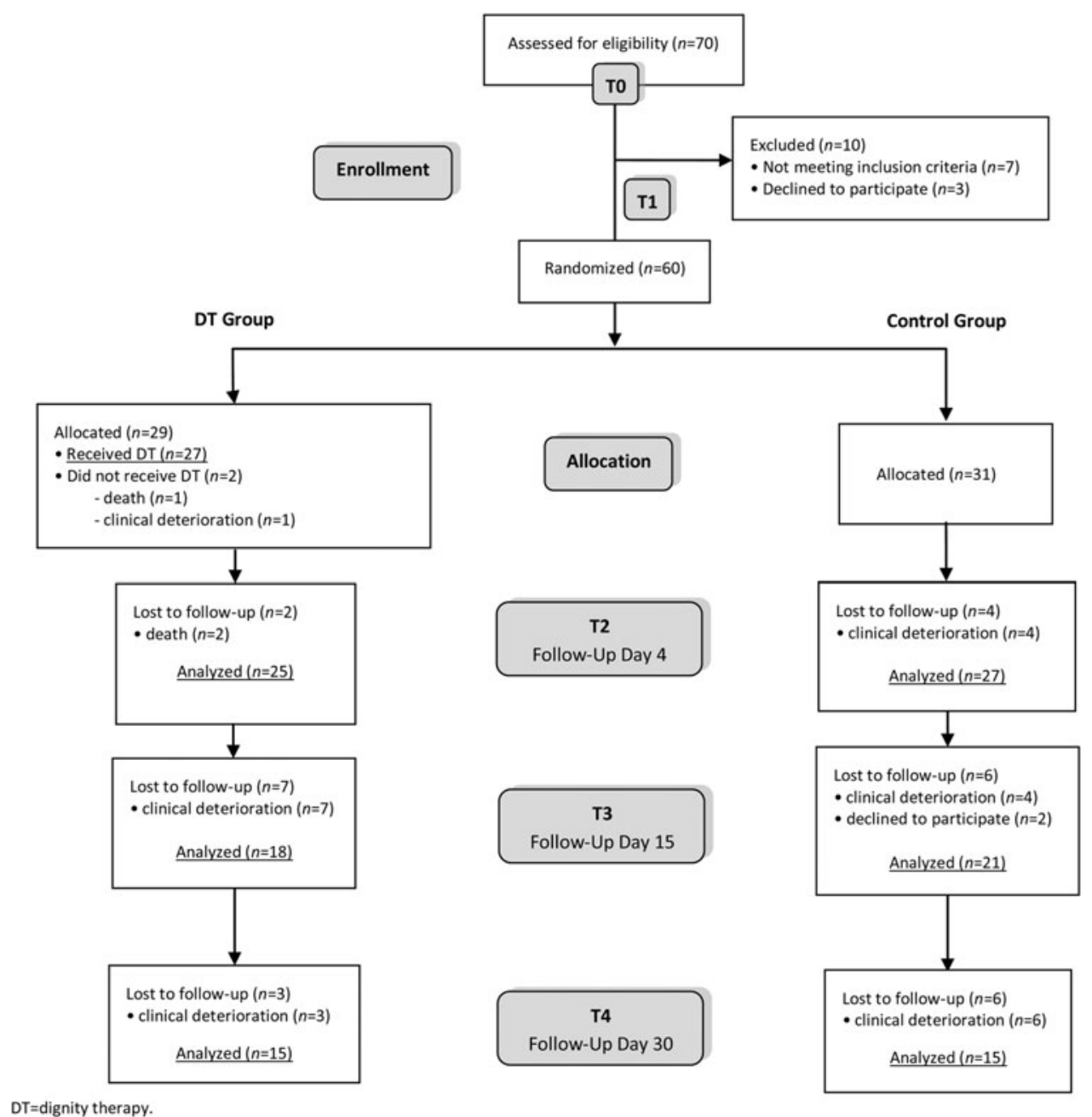

Fig. 1. Consort diagram. 
Table 4. Primary efficacy outcome variable: HADS depression subscale score within and between study groups

\begin{tabular}{|c|c|c|c|}
\hline & & Control group & DT group \\
\hline \multirow[t]{2}{*}{$\mathrm{T} 2$} & $\begin{array}{l}\text { Difference from baseline } \\
n \\
\text { mean }(95 \% \mathrm{CI}) \\
p \text { value }\end{array}$ & $\begin{array}{c}27 \\
1.70(0.35,3.01) \\
0.016^{\mathrm{a}} ; 0.012^{\mathrm{b}}\end{array}$ & $\begin{array}{c}25 \\
-2.76(-4.86,-0.66) \\
0.012^{\mathrm{a}} ; 0.010^{\mathrm{b}}\end{array}$ \\
\hline & $\begin{array}{l}\text { Difference between groups } \\
\text { mean }(95 \% \mathrm{CI}) \\
p \text { value }\end{array}$ & & $\begin{array}{c}-4.46(-6.91,-2.02) \\
0.001^{\mathrm{c}} ;<0.0001^{\mathrm{d}}\end{array}$ \\
\hline \multirow[t]{2}{*}{$\mathrm{T} 3$} & $\begin{array}{l}\text { Difference from baseline } \\
n \\
\text { mean }(95 \% \mathrm{CI}) \\
p \text { value }\end{array}$ & $\begin{array}{c}21 \\
2.52(1.02,4.03) \\
0.002^{\mathrm{a}} ; 0.003^{\mathrm{b}}\end{array}$ & $\begin{array}{c}18 \\
-1.44(-4.53,1.64) \\
0.034^{\mathrm{a}} ; 0.367^{\mathrm{b}}\end{array}$ \\
\hline & $\begin{array}{l}\text { Difference between groups } \\
\text { mean }(95 \% \mathrm{CI}) \\
p \text { value }\end{array}$ & & $\begin{array}{c}-3.96(-7.33,-0.61) \\
0.022^{\mathrm{c}} ; 0.062^{\mathrm{d}}\end{array}$ \\
\hline \multirow[t]{2}{*}{$\mathrm{T} 4$} & $\begin{array}{l}\text { Difference from baseline } \\
n \\
\text { mean }(95 \% \mathrm{CI}) \\
p \text { value }\end{array}$ & $\begin{array}{c}15 \\
3.40(1.66,5.14) \\
0.001^{\mathrm{a}} ; 0.002^{\mathrm{b}}\end{array}$ & $\begin{array}{c}15 \\
0.06(-3.65,3.78) \\
0.970^{\mathrm{a}} ; 0.801^{\mathrm{b}}\end{array}$ \\
\hline & $\begin{array}{l}\text { Difference between groups } \\
\text { mean }(95 \% \mathrm{CI}) \\
p \text { value }\end{array}$ & \multicolumn{2}{|r|}{$\begin{array}{c}-3.33(-7.32,0.65) ; \\
0.097^{\mathrm{c}} ; 0.173^{\mathrm{d}}\end{array}$} \\
\hline
\end{tabular}

Evaluation time points: $\mathrm{T} 2=$ post-intervention; $\mathrm{T} 3=$ day $15 ; \mathrm{T} 4=$ day 30 .

${ }^{\text {a }}$ Paired $t$ test.

b Wilcoxon test.

c independent $t$ test.

d Mann-Whitney test.

CI, confidence interval; DT, dignity therapy; HADS, Hospital Anxiety \& Depression Scale.

post-intervention and day 15 in the control group. Results obtained by the nonparametric tests confirmed the parametric test findings, with the exception of day 30 (Table 4).

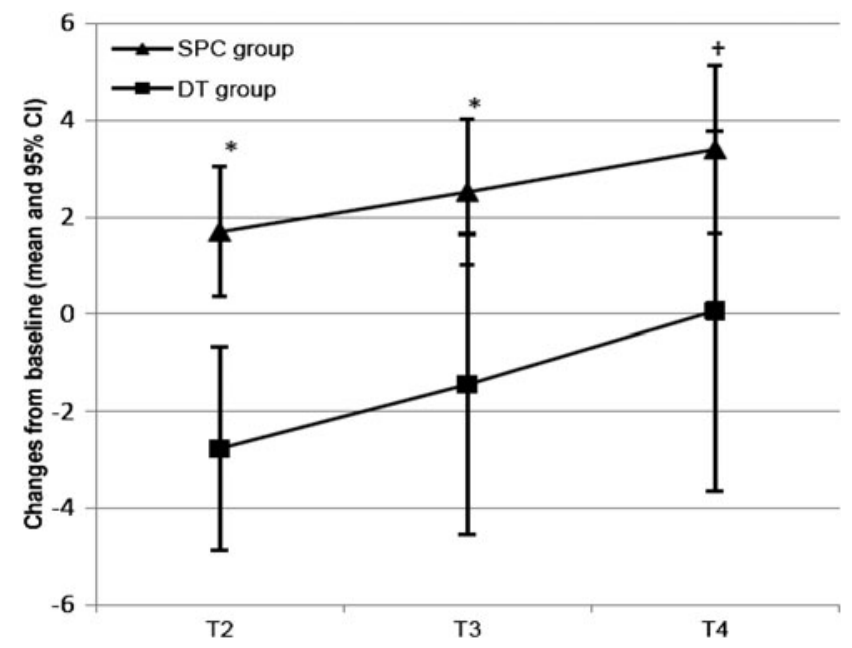

Fig. 2. Changes from baseline on HADS depression subscale mean score. $\mathrm{DT}=$ dignity therapy; $\mathrm{SPC}=$ standard palliative care; HADS $=$ Hospital Anxiety \& Depression Scale. T2 = day 4; $\mathrm{T} 3=$ day 15 ; T4 $=$ day $30 .{ }^{*}$ Independent $t$ test, $p<0.05 .{ }^{\dagger}$ Independent $t$ test, $p=0.097$.

\section{Anxiety}

Both groups had a mean score (SD) on the HADS anxiety subscale $<11$ at baseline (SPC:8.40 [4.55]; DT:8.83 [4.97]; $p=0.731$ ).

Compared with the SPC group, those receiving DT demonstrated significantly lower anxiety ratings at all assessment periods (post-intervention: mean $=-3.96,95 \% \mathrm{CI},-6.66$ to $-1.25, p=0.005$; day 15 : mean $=-6.19,95 \% \mathrm{CI},-10.49$ to -1.88 , $p=0.006$; day 30 : mean $=-5.07,95 \% \mathrm{CI},-10.22-$ $0.09, p=0.054$ ) (Table 5).

Within the DT group, there was a significant reduction on anxiety ratings between baseline and day 4 assessment $($ mean $=-3.44,95 \% \mathrm{CI},-5.42$ to $-1.46, p=0.001$ ), which was maintained throughout the follow-up period (day 15: mean $=-4.33,95 \% \mathrm{CI}$, -7.68 to $-0.99, p=0.014$; day 30 : mean $=-4.07$, $95 \%$ CI, -7.79 to $-0.34, p=0.034$ ) (Table 5, Fig. 3).

In the SPC group, there were no significant changes on the HADS anxiety subscale between baseline and day 4, or throughout the subsequent follow-ups $($ mean $=0.52, \quad 95 \% \quad$ CI $, \quad-1.42-2.46, \quad p=0.588$; mean $=1.86,95 \% \quad$ CI, $-1.08-4.79, p=0.202$; mean $=1.00, \quad 95 \% \quad$ CI $, \quad-2.91-4.91, \quad p=0.591$ ) (Table 5). 
Table 5. Primary efficacy outcome variable: HADS anxiety subscale score within and between study groups

\begin{tabular}{|c|c|c|c|}
\hline & & Control group & DT group \\
\hline \multirow[t]{2}{*}{$\mathrm{T} 2$} & $\begin{array}{l}\text { Difference from baseline } \\
n \\
\text { mean }(95 \% \mathrm{CI}) \\
p \text { value }\end{array}$ & $\begin{array}{c}27 \\
0.52(-1.42,2.46) \\
0.588^{\mathrm{a}} ; 0.146^{\mathrm{b}}\end{array}$ & $\begin{array}{c}25 \\
-3.44(-5.42,-1.46) \\
0.001^{\mathrm{a}} ; 0.002^{\mathrm{b}}\end{array}$ \\
\hline & $\begin{array}{l}\text { Difference between groups } \\
\text { mean }(95 \% \mathrm{CI}) \\
p \text { value }\end{array}$ & & $\begin{array}{c}-3.96(-6.66,-1.25) \\
0.005^{\mathrm{c}} ; 0.001^{\mathrm{d}}\end{array}$ \\
\hline \multirow[t]{2}{*}{$\mathrm{T} 3$} & $\begin{array}{l}\text { Difference from baseline } \\
n \\
\text { mean }(95 \% \mathrm{CI}) \\
p \text { value }\end{array}$ & $\begin{array}{c}21 \\
1.86(-1.08,4.79) \\
0.202^{\mathrm{a}} ; 0.191^{\mathrm{b}}\end{array}$ & $\begin{array}{c}18 \\
-4.33(-7.68,-0.99) \\
0.014^{\mathrm{a}} ; 0.015^{\mathrm{b}}\end{array}$ \\
\hline & $\begin{array}{l}\text { Difference between groups } \\
\text { mean }(95 \% \mathrm{CI}) \\
p \text { value }\end{array}$ & & $\begin{array}{c}-6.19(-10.49,-1.88) \\
0.006^{\mathrm{c}} ; 0.007^{\mathrm{d}}\end{array}$ \\
\hline \multirow[t]{2}{*}{$\mathrm{T} 4$} & $\begin{array}{l}\text { Difference from baseline } \\
n \\
\text { mean }(95 \% \mathrm{CI}) \\
p \text { value }\end{array}$ & $\begin{array}{c}15 \\
1.00(-2.91,4.91) \\
0.591^{\mathrm{a}} ; 0.555^{\mathrm{b}}\end{array}$ & $\begin{array}{c}15 \\
-4.07(-7.79,-0.34) \\
0.034^{\mathrm{a}} ; 0.025^{\mathrm{b}}\end{array}$ \\
\hline & $\begin{array}{l}\text { Difference between groups } \\
\text { mean }(95 \% \mathrm{CI}) \\
p \text { value }\end{array}$ & \multicolumn{2}{|r|}{$\begin{array}{c}-5.07(-10.22,0.09) \\
0.054^{\mathrm{c}} ; 0.042^{\mathrm{d}}\end{array}$} \\
\hline
\end{tabular}

Evaluation time points: T2 = post-intervention; $\mathrm{T} 3=$ day $15 ; \mathrm{T} 4=$ day 30 .

${ }^{\text {a }}$ Paired $t$ test.

b Wilcoxon test.

${ }^{\mathrm{c}}$ Independent $t$ test.

d Mann-Whitney test.

CI, confidence interval; DT, dignity therapy; HADS, Hospital Anxiety \& Depression Scale.

Normality assumption was not rejected for any of the time measures using the HADS anxiety subscale. Nonetheless, nonparametric tests were also applied, confirming the $t$ test findings (Table 5).

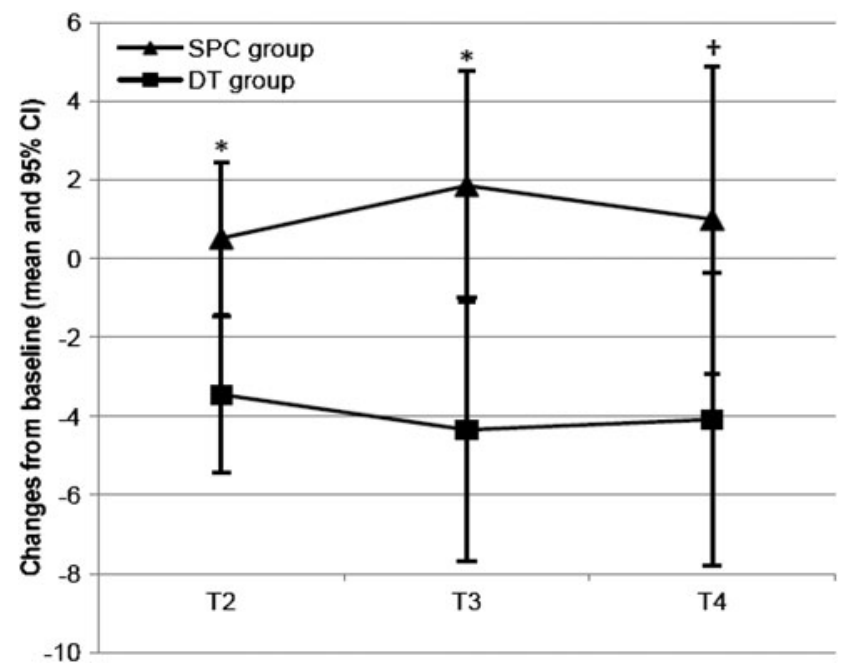

Fig. 3. Changes from baseline on HADS anxiety subscale mean score. $\mathrm{DT}=$ dignity therapy. $\mathrm{SPC}=$ standard palliative care. HADS $=$ Hospital Anxiety \& Depression Scale. T2 = day 4; T3= day $15 ; \mathrm{T} 4=$ day $30 .{ }^{*}$ Independent $t$ test, $p<0.05$. Independent $t$ test, $p=0.054$.

\section{DISCUSSION}

As previously demonstrated in the Portuguese cancer population (Pais-Ribeiro et al., 2007), our data evidence a high level of depression and anxiety symptoms at baseline. DT research has been shown to enhance end-of-life experience, but has not as yet been shown to ameliorate depression and anxiety symptoms in more severely distressed patients such as our patient sample. In fact, low base rates of distress were posited as an explanation for the inability to demonstrate DT's influence on depression or anxiety in prior clinical trials (Chochinov, 2011). On that front, this Portuguese cohort was clearly more distressed at baseline than that in the Chochinov et al. study (mean HADS-depression $=12.9$ versus 5.86 ; mean HADS-anxiety $=8.83$ versus 5.22 , respectively).

Patients who received DT were significantly more likely than SPC patients to experience reductions in depression ratings immediately post-treatment, with improvement being sustained throughout subsequent follow-up, with the exception of depression on day 30. The lack of efficacy of DT on reducing HADS-depression scores at day 30 could suggest that one single DT session might not be enough to ameliorate and maintain the reductions in 
depressive symptoms of patients facing death. These findings beg the question of whether a second followup session might reinforce the initial improvements yielded by DT. Future research exploring this therapeutic approach would be of great value.

Even though our patient population had a high level of psychosocial distress, DT was offered to every eligible participant without considering the presence or intensity of psychological distress at baseline. Our results show that patients with high levels of distress can perform DT without distortion or misrepresentation of their life narratives.

Similar findings were demonstrated in terms of DT's ability to mitigate anxiety. These findings are notable, given that Chochinov and colleagues' recent trial (Chochinov et al., 2011) was unable to demonstrate a significant effect of DT on anxiety. Low base rates of distress were posited as an explanation for the inability to demonstrate DT's influence on this outcome. By allowing patients to remember, speak about their past, and share important reflections, DT brings these special moments to the present, reaffirming a sense of meaning and purpose. The therapeutic process, along with the opportunity to create a personal generativity document, offers patients the opportunity to actively engage in a vital role, diminishing their sense of helplessness and despair.

Perhaps patients who engage in DT are able to move beyond their sadness, to an activity imbued with meaning and purpose. It may also be that as patients focused on their responses, experiencing a degree of distraction that manifested itself as a reduction of anxiety; although it is interesting to note that this effect was sustained well beyond the time of therapeutic engagement.

There are several limitations to this study worth noting. As in other published DT studies (Chochinov et al., 2002; 2005; 2011) our research took place primarily among older patients. As such, the influence of DT within younger cohorts has yet to be established. We also acknowledge that the study sample size was small, which speaks for the reality of doing longitudinal studies in very sick palliative care populations. In spite of this limitation, there are substantial between-group statistical differences, indicating clinically significant improvement. Finally, the majority of the study participants had end-stage malignancies. Future research may explore the utility of DT for other terminal conditions, such as end-stage organ failure diseases. Within this trial, DT was performed exclusively by the principal investigator (MJ), who strictly followed the DT protocol (Chochinov, 2011) and received training provided by the originators of this therapeutic approach. The DT therapist was not blinded to the study arm assign- ment, however, patients in both groups received equal quality palliative care (SPC) and all evaluations, treatment plans, and direct interventions were conducted, as per unit protocol, by the entire multidisciplinary team. Despite these limitations, this study provides compelling support for the efficacy of DT in addressing symptoms of anxiety and depression among patients.

Dignity therapy appears to have short-term benefits on the psychological distress encountered by patients near the end of life. This has important future clinical and research implications; DT should now be evaluated relative to other treatment options such as psychotherapy or the use of psychopharmaceuticals.

\section{CONTRIBUTORS}

$\mathrm{MJ}$ and $\mathrm{AB}$ were responsible for the conception and design. MJ was responsible for supervising the study, analyzing the data, and writing the initial draft and final report. FO was responsible for the scales assessments. BN supervised the data statistical analysis. AVC helped with the revising of the final report. All co-authors helped in revising the final document and had full access to all of the data.

\section{ACKNOWLEDGMENTS}

The authors thank Filipa Fareleira for constant and pertinent revision of the report's scientific content, Vivelinda Guerreiro and Sofia Mata for general administrative support, as well as the bibliography research, João Costa for his help on the consolidation of the initial protocol, Carlos Centeno for supporting this project, Todd Weeks for helping to revise and correct the English grammar, and Professor Chochinov for the guidance and wisdom he shared periodically during the course of this study.

\section{REFERENCES}

Block, S.D. (2000). Assessing and managing depression in the terminally ill patient. ACP-ASIM End-of-Life Care Consensus Panel. American College of Physicians American Society of Internal Medicine. Annals of Internal Medicine, 132, 209-218.

Boston, P., Bruce, A. \& Schreiber, R. (2011). Existential suffering in the palliative care setting: An integrated literature review. Journal of Pain and Symptom Management, $41,604-618$.

Chochinov, H.M. (2007). Dignity and the essence of medicine: The A, B, C, and D of dignity conserving care. British Medical Journal, 335, 184-187.

Chochinov, H.M. (2011). Dignity Therapy: Final Words for Final Days. New York: Oxford University Press.

Chochinov, H.M., Hack, T., Hassard, T., et al. (2005). Dignity therapy: A novel psychotherapeutic intervention for patients near the end of life. Journal of Clinical Oncology, 23, 5520-5525. 
Chochinov, H.M., Hack, T., McClement, S., et al. (2002). Dignity in the terminally ill: A developing empirical model. Social Science in Medicine, 54, 433-443.

Chochinov, H.M., Kristjanson, L.J., Breitbart, W., et al. (2011). Effect of dignity therapy on distress and end-oflife experience in terminally ill patients: A randomised controlled trial. Lancet Oncology, 12, 753-762.
Chochinov, H.M., Wilson, K.G., Enns, M., et al. (1995). Desire for death in the terminally ill. American Journal of Psychiatry, 152, 1185-1191.

Pais-Ribeiro, J., Silva, I., Ferreira, T., et al. (2007). Validation study of a Portuguese version of the Hospital Anxiety and Depression Scale. Psychology, Health \& Medicine, 12, 225-235. 